\title{
Tunable Violet Laser Diode System for Optical Wireless Communication
}

\author{
Sani Mukhtar, Sun Xiaobin, Islam Ashry Senior Member, IEEE, Tien Khee Ng, Senior Member, \\ IEEE, Boon S. Ooi, and M. Z. M. Khan, Senior Member, IEEE
}

\begin{abstract}
- we report a tunable self-injection locked violet laser diode external cavity system exhibiting a continuous wavelength tunability of $5.15 \mathrm{~nm}(400.28-405.43 \mathrm{~nm})$ with mean side-modesuppression-ratio (SMSR) and linewidth of $\sim 23 \mathrm{~dB}$ and $\sim 190 \mathrm{pm}$, respectively. The effects of injection current and temperature indicate a robust system besides being cost-effective and straightforward. Moreover, a successful indoor on-off keying transmission at two different locked modes on a $0.4 \mathrm{~m}$ free space channel showed $\sim 10$ times improvement in the bit-error-rate (BER) with value $\sim 8 \times 10^{-4}$ at $2 \mathrm{~Gb} / \mathrm{s}$, and better performance on $0.8 \mathrm{~m}$ channel length at $1.75 \mathrm{~Gb} / \mathrm{s}$ compared to the free-running laser case. Our work is a potential step towards the realization of future high data capacity narrow-wavelength-spaced multiplexed optical wireless communication system wherein continuously tunable laser sources are expected to play a crucial role as transmitters.
\end{abstract}

Index Terms - violet laser, tunable laser, external cavity systems, self-injection locking, optical wireless communication

\section{INTRODUCTION}

$\mathrm{O}$ ptical wireless communication (OWC) remained mostly unexplored until recently, has taken center stage, thanks to the advent of visible laser diodes (LDs), emerging visible light communication (VLC), and underwater optical wireless communication (UOWC). Moreover, the inherent limitations in the radio frequency (RF) spectrum, added with higher secured end-user data requirements, low power consumption,

Manuscript received XXX XX, XXX; revised XXXXXX X, XXXX; accepted XXXXXX XX, XXXX. Date of publication XXXXXX XX, XXX; date of current version XXXXXX XX, XXXX. Authors at King Fahd University of Petroleum and Minerals (KFUPM) acknowledge the support received from King Abdulaziz City for Science and Technology (KACST), Saudi Arabia, through grant no. EE2381 that is sub-awarded from the main grant no. KACST TIC R2-FP-008. Authors from King Abdullah University of Science and Technology (KAUST), acknowledge the funding (grant nos. BAS/1/161401-01, KCR/1/2081-01-01, and GEN/1/6607-01-01); and KAUST-KFUPM Special Initiative (KKI) Program (REP/1/2878-01-01).(Corresponding author: M. Z. M. Khan)

Sani Mukhtar and M. Z. M. Khan are with the Optoelectronic Research Laboratory (ORL), Electrical Engineering Department, KFUPM, Dhahran 31261, Saudi Arabia. (e-mail: g201802620@kfupm.edu.sa and zahedmk@kfupm.edu.sa)

Sun Xiaobin, Islam Ashry, Tien K. Ng and B. S. Ooi are with Photonics Laboratory, Computer, Electrical and Mathematical Sciences and Engineering (CEMSE) Division, KAUST, Thuwal 23955-6900, Saudi Arabia. (e-mail: sun.xiaobin@kaust.edu.sa; tienkhee.ng@kaust.edu.sa; boon.ooi@kaust.edu.sa)

Color versions of one or more of the figures in this letter are available online at http://ieeexplore.ieee.org.

Digital Object Identifier 10.1109/LPT.XXXXXXXXXX etc.[1][2][3] have prompted exploring VLC and its subset LiFi (light fidelity) for short-reach communication as well as last-mile access solution, seamlessly integrating with the existing optical fiber network infrastructure and hence complementing the present Wi-Fi (wireless fidelity) technology [4]. Moreover, visible wavelength division multiplexing (WDM) is also gaining interest where wide wavelengths spaced red $(\sim 630 \mathrm{~nm})$ - blue $(\sim 430 \mathrm{~nm})$ - green $(\sim 530 \mathrm{~nm})$ colors are employed as subcarrier channels to increase the OWC system data capacity[5].

Accordingly, InGaN/GaN blue-violet semiconductor LDs are widely employed not only in VLC but also in UWOC due to their least absorption in water. However, their inherent broad lasing emission exhibiting several longitudinal modes poses restriction to this Fabry-Perot resonator-based devices in terms of direct-modulation capability, signal-to-noise-ratio (SNR) and hence the transmitted data rate. A LD with narrow optical linewidth, high SMSR and SNR, and therefore large modulation bandwidth, is preferred in these applications. Additionally, the possibility of tuning the narrow emission wavelength of blue-violet LD would not only unlock its potential deployment in various multidisciplinary field applications such as high-resolution spectroscopy [6], sensing [7], etc. but also enable the realization of future narrow wavelength spaced blue-violet color WDM-OWC system. Moreover, integration of multiplexed blue-violet color with red and green color for future LD based large data capacity LiFi systems would be the next potential step, thus optimizing the available unregulated optical bandwidth of visible region.

To achieve narrow optical linewidth and wavelength tunability in the blue-violet region, the external cavity diode laser (ECDL) system has been the most conventional approach in literature besides other techniques, and their performances are summarized in Table 1. As noted from the table, the largest tuning window of $6.3 \mathrm{~nm}$ centered at $\sim 400 \mathrm{~nm}$ is reported in [8] with linewidth $<11 \mathrm{MHz}$ utilizing a Littrow ECDL system with a wavelength grating filter. However, complexity, misalignment, and instability at higher injection current are some of the limitations of these systems besides being costly. Hence, a robust and simple tuning scheme is preferred for practical applications. Additionally, hydrostatic pressure has also been employed to tune the wavelength from $\sim 406.5-416 \mathrm{~nm}(9.5 \mathrm{~nm})$ in [9], but the optical linewidth was very broad with multiple longitudinal modes.

Very recently, a tunable visible LD system with assistive self-injection locking (SIL) scheme stands out as a competing candidate to obtain spectral purity and wavelength tunability, 
TABLE I

ADVANCEMENT IN TUNABLE VIOLET LASER DIODES AND THEIR PERFORMANCE IN OWC

\begin{tabular}{|c|c|c|c|c|c|c|c|c|c|}
\hline Wavelength (nm) & $\Delta W(\mathrm{~nm})$ & $\mathrm{TM}$ & SMSR (dB) & $\Delta \lambda(\mathrm{pm})$ & $C_{L}(\mathrm{~m})$ & $D(\mathrm{~Gb} / \mathrm{s})$ & Scheme & BER & Ref. \\
\hline $395.60-401.90$ & 2.7 & $\mathrm{~L}$ & - & $5 \mathrm{MHz}$ & - & - & - & - & [14] \\
\hline $397.20-403.50$ & 6.3 & LM & - & $11 \mathrm{MHz}$ & - & - & - & - & [8] \\
\hline $396.45-400.20$ & 5.5 & $\mathrm{~L}$ & - & - & - & - & - & - & [15] \\
\hline $404.25-405.00$ & 0.8 & HG & 15 & 200 & - & - & - & - & [16] \\
\hline $403.82-408.29$ & 4.4 & $\mathrm{~L}$ & 20 & 100 & - & - & - & - & [17] \\
\hline $404.11-410.12$ & 6.0 & $\mathrm{~L}$ & - & 30 & - & - & - & - & [18] \\
\hline 405 & & & - & - & $4.8^{\mathrm{UW}}$ & 1.45 & 16-QAM OFDM & $9.1 e^{-4}$ & [19] \\
\hline 404 & & & - & - & $7.0^{\mathrm{FS}}$ & 12 & 16-QAMOFDM & $3.8 e^{-3}$ & [1] \\
\hline 410 (RGB) & & & & & $0.15^{\mathrm{FS}}$ & 1.5 & OOK NRZ & $1.8 e^{-3}$ & [2] \\
\hline 405 & & & - & - & $0.5^{\mathrm{FS}}$ & 26.4 & 64-QAM DMT & $3.17 e^{-3}$ & [3] \\
\hline $400.28-405.4$ & 5.1 & SIL & $>16$ & & $\begin{array}{l}0.4^{\mathrm{FS}} \\
0.8^{\mathrm{FS}}\end{array}$ & $\begin{array}{c}2 \\
1.75 \\
\end{array}$ & $\begin{array}{l}\text { OOK NRZ } \\
\text { OOK NRZ }\end{array}$ & $\begin{array}{l}8 e^{-4} \\
3 e^{-3} \\
\end{array}$ & $\begin{array}{l}\text { This } \\
\text { Work }\end{array}$ \\
\hline
\end{tabular}

$\Delta w$. Tuning span, TM: Tuning Method, L: Littrow, LM: Littman, HG: Holographic grating, $\Delta \lambda$ : Linewidth, $\mathrm{C}_{L}$ : Channel length, FS: Free Space, UW: Underwater, $D$ : Data rate, RGB: Red-blue-green multiplexed system

while being compact and cost-effective [10]. Moreover, such a configuration would be a promising candidate for the realization of future narrow wavelength spaced WDM-VLC as well as WDM-UWOC systems and easily integrated with the existing optical access infrastructure. To this end, Table 1 also summarizes the advances in single-channel OWC employing a free-running (FR) violet $\mathrm{LD}$ with the largest reported data rates of 1.5 and $26.4 \mathrm{~Gb} / \mathrm{s}$ using on-off keying (OOK) and quadrate amplitude modulation (64-QAM) schemes, respectively. Employing a narrow optical linewidth $\mathrm{LD}$ in OWC, with added tunability feature, is expected to improve the transmission characteristics further while providing wavelength flexibility, which is crucial for eventual visible WDM systems.

In this letter, we report a self-injection locked ECDL (SILECDL) system for a violet LD, where a continuous wavelength tunability of $\Delta w=5.15 \mathrm{~nm}$ with average SMSR and $\Delta \lambda$ of $\sim 23 \mathrm{~dB}$ and $\sim 190 \mathrm{pm}$, respectively, have been achieved. An error-free indoor OOK transmission on $0.4 \mathrm{~m}$ channel length is demonstrated on two widely tuned wavelengths of 401.14 and $403.18 \mathrm{~nm}$ with a realized data rate of $2.0 \mathrm{~Gb} / \mathrm{s}$ showing superior performance compared to the FR case. Moreover, extending the channel length to $0.8 \mathrm{~m}$ depicted similar transmission characteristics with a maximum data rate of $1.75 \mathrm{~Gb} / \mathrm{s}$. This work further strengthens the prospects of compact tunable laser diode system for future multiplexed VOWC systems as well as for cross-disciplinary field applications.

\section{EXPERIMENTAL SETUP}

The schematic block diagram of the tunable laser system is illustrated in Fig. 1. A commercial low power $405 \mathrm{~nm}$ violet laser (Thorlabs, DL5146-101S) is fixed on a temperaturecontrolled laser diode mount (Thorlabs, TCLDM9) with designated $1 \mathrm{GHz}$ bandwidth. A pellicle beam splitter (BS1) with 92:8\% splitting ratio (Thorlabs, BP107) is employed within the external cavity, where the transmitted $92 \%$ of the optical power is passed through a prism P (Thorlabs, PS858) and then reflected back into the laser front facet using a silvercoated mirror with $97.5 \%$ reflection (Thorlabs, PF10-03-P01). The distance between the laser front facet and the mirror forms the external cavity whose length is fixed at $17 \mathrm{~cm}$. The reflected $8 \%$ of the usable optical power from the tunable system is further split by BS2 (Thorlabs, BSW27) with 50\% falling on the Photodiode PD (Thorlabs, APD210, $1 \mathrm{GHz}$ bandwidth) and the other 50\% going into an optical spectrum analyzer (Yokogawa, AQ6373B, 20 pm resolution) after each being collimated with plano-convex lens (Thorlabs, LA-1951A) L2 and L3, respectively. An indoor free space channel length of 0.4 or $0.8 \mathrm{~m}$ is formed between the tunable source (i.e., from BS2) and the photodiode. For the transmission experiments, a $0.9 \mathrm{~V}$ OOK non-return to zero (OOK-NRZ) signal is generated from a pattern generator (Keysight N4903B) with pseudorandom binary sequence (PRBS) length $2^{10}-1$, and used to modulate the LD (intensity modulation, IM), and then the received signal after the photodiode is analyzed via direct detection (DD) configuration on digital communication analyzer (DCA, Agilent DCA-J 86100C) and a bit error-rate-tester (BERT, Keysight N4903B). The dynamic range of laser biasing is selected above the threshold, with modulation index $\sim 0.17$. A fully reflecting mirror mounted on flip Mount (Thorlabs, TRF90/M) is placed within the external cavity to misalign the optical feedback beam during the transmission experiments for performance comparison between the FR and the locked mode lasing spectrums, and at identical current and temperature operation.

\section{TheORETICAL Discussion}

Let the phase of a particular LD longitudinal mode under FR and under optical feedback be $\phi_{f}$ and $\phi_{o}$, respectively, and the phase provided by the external cavity be $\phi_{c o}$. Then, the

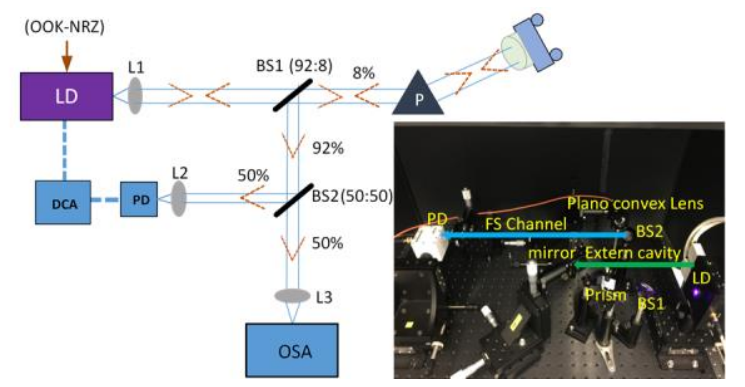

Fig. 1. The Schematic diagram of the SIL violet laser diode system with integrated transmission module. The inset show the photograph of the laboratory setup 
conditional relation of the LD's phase under optical injection can be given as follows [11]:

$$
\phi_{o}=\alpha \sqrt{\left(1+\beta^{2}\right)} \sin \left(\phi_{c o}+\tan ^{-1} \beta\right)+\phi_{f}
$$

where $\alpha$ is the optical injection coefficient, which is a function of feedback optical power coupling efficiency, $\beta$ is the linewidth enhancement factor of the LD that depends on the change in refractive index due to the change in gain threshold. Now, $\phi_{c o}$ can be steered by tuning the external cavity length to obtain matching phase condition $\left(\phi_{o}=\phi_{f}\right.$ or multiple of $\left.2 \pi\right)$ to assure this particular LD mode resonates in the two-cavity system, giving rise to a locked mode with a reduced gain threshold. Thus, allowing this locked mode to reach lasing condition earlier than the other modes, and hence dominates by suppressing all the adjacent modes. This phenomenon leads to the enhancement in $\Delta \lambda$, SMSR of the dominant mode, and, consequently, the spectral purity of the laser with reduced amplified spontaneous emission (ASE) noise and high SNR [12][13]. Moreover, altering the external cavity with solutions to any LD mode could be attained, thus tuning the locked mode wavelength within the gain profile of the LD active region.
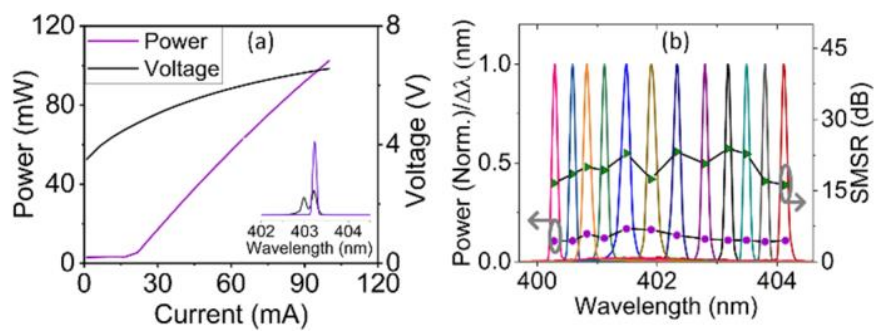

Fig. 2. (a) Room temperature L-I-V characteristics of the violet InGaN/GaN LD with the comparison between the FR and SIL spectrum at $25 \mathrm{~mA}$ in the inset. (b) Room temperature wavelength tunability of the violet tunable SILECDL system at $21 \mathrm{~mA}$ alongside the corresponding extracted $\Delta \lambda$ and the SMSR.

\section{WAVELENGTH TUNABILITY CHARACTERIZATION}

First, the front single facet L-I-V performance of the FR violet LD is obtained, as shown in Fig. 2(a), exhibiting a threshold current $I_{t h}=20 \mathrm{~mA}$ and a near-threshold slope efficiency of $1.2 \mathrm{~W} / \mathrm{A}$ at room temperature. A comparison between the SIL mode and the FR spectrum is illustrated in the inset of Fig. 2(a) demonstrating a significant improvement by $\sim 3.0, \sim 16.5$, and $\sim 3.1$ times in $\Delta \lambda$, SMSR, and the peak power, respectively. Thanks to the SIL scheme that enabled this profound improvement besides improving the modulation bandwidth and the noise of the LD [12][13]. The tunability of the system is accessed at three injection currents of 21 $\left(\sim 1.0 I_{t h}\right), 42\left(\sim 2.0 I_{t h}\right)$, and $63 \mathrm{~mA}\left(\sim 3.0 I_{t h}\right)$, and two distinct temperatures of 20 (room temperature) and $40^{\circ} \mathrm{C}$. A continuous wavelength tuning of the locked modes satisfying Eqn. 1 is achieved by fine-tuning the external cavity length and the optical feedback angle manually. The room temperature results of the tuning span at $\sim 1.0 I_{t h}$ are summarized in Fig. 2(b) with the corresponding extracted $\Delta \lambda$ and SMSR. As shown, a wavelength tuning of $3.84 \mathrm{~nm}$ (400.28 to $404.12 \mathrm{~nm}$ ) is achieved, exhibiting a minimum (maximum) $\Delta \lambda$ of 106 (168) $\mathrm{pm}$ while the SMSR is
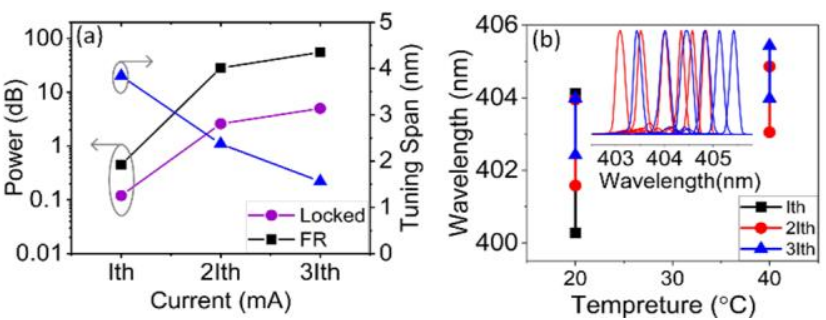

Fig. 3. (a) Comparison of the usable optical power of the violet tunable SILECDL system with the FR LD power at three different injection currents along with the corresponding measured wavelength tuning window. (b) Temperature effect on the wavelength span of the violet tunable SIL-ECDL system at different injection currents. The inset of (b) depicts the normalized locked spectrums tunability at various current injections, and at $40^{\circ} \mathrm{C}$.

maintained at $>16 \mathrm{~dB}$ (highest $24 \mathrm{~dB}$ ) in the entire tuning range. However, on increasing the injection current to $\sim 2.0 I_{t h}$ and $\sim 3.0 I_{t h}$, the tuning window drops to $2.37 \mathrm{~nm}$ (401.58 to $403.95 \mathrm{~nm})$ and $1.56 \mathrm{~nm}(402.42$ to $403.98 \mathrm{~nm})$, respectively, as depicted in Fig. 3(a). A similar trend is also observed on increasing the temperature to $40^{\circ} \mathrm{C}$, as shown in Fig 3(b). In this case, the tuning span reduced to $1.77 \mathrm{~nm}$ (403.09 to $404.86 \mathrm{~nm})$ and $1.45 \mathrm{~nm}(403.98$ to $405.43 \mathrm{~nm})$ at $\sim 2.0 I_{\text {th }}$ and $\sim 3.0 I_{t h}$, respectively, with associated mean $\Delta \lambda$ and SMSR values of $\sim 186 \mathrm{pm}$ and $\sim 15 \mathrm{~dB}$, and $\sim 230 \mathrm{pm}$ and $\sim 20 \mathrm{~dB}$, respectively. In general, the overall tunability of the system degraded at a higher temperature at any injection current. This inferior performance at high injection currents and temperature is attributed to the dynamic behavior of the optical feedback system, which supplements mode competition in a bid to satisfy Eqn. 1 as well as the change in $\beta$ that is injection current and temperature dependent. It is worth mentioning at this instant that the system exhibits an overall tunability of $5.15 \mathrm{~nm}(400.28$ to $405.43 \mathrm{~nm})$ should temperature and injection current are considered as a tuning parameter. Moreover, employing a prism within the external cavity enhances the system performance and stability, which is ascribed to improved locking efficiency (effectively satisfying Eqn. 1) by slightly dispersing the optical feedback modes wavelength, thereby reducing their encounter with a LD mode in satisfying Eqn. 1. The usable optical power of the tunable SIL-ECDL system is compared with the FR LD power in Fig. 3(a) at room temperature and different injection currents. As expected, the output power of the system is approximately 8.0 $\%$ of the FR power, reaching from $0.12 \mathrm{~mW}$ at $\sim 1.0 I_{\text {th }}$ to 5.0 $\mathrm{mW}$ at $\sim 3.0 I_{t h}{ }^{\prime}$ while the corresponding measured FR LD powers are $0.45 \mathrm{~mW}$ and $55 \mathrm{~mW}$. Moreover, it is to be noted that if the rear as-cleaved facet of the LD is accessible, then $\sim 70-80 \%$ of the FR power would be available as the working
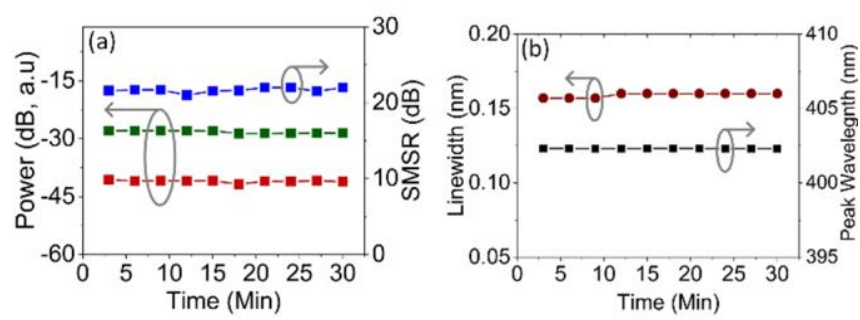

Fig. 4 Stability performance of the violet tunable SIL-ECDL system at a tuned wavelength of $402.29 \mathrm{~nm}$ in terms of (a) SMSR (blue), integrated power (green) and peak power (red), and (b) optical linewidth and peak wavelength. 
power.

Lastly, a stability check of the system is performed by monitoring the peak wavelength, peak power, SMSR and $\Delta \lambda$ of $402.29 \mathrm{~nm}$ locked mode for $30 \mathrm{~min}$ with a step size of 3 min, and the results are plotted in Fig. 4. The integrated and peak powers displayed a variation of $\pm 0.25 \mathrm{~dB}$ while the SMSR remained at $\pm 0.5 \mathrm{~dB}$, as depicted in Fig. 4(a). On the other hand, from Fig. 4(b), the peak wavelength fluctuation of $\pm 4.0 \mathrm{pm}$, and $\Delta \lambda$ variation of $\pm 1.5 \mathrm{pm}$ are noted. These performance indicators exhibiting minimal deviation affirm the high stability of the system and its potential for practical deployment.

\section{Visible Optical WiRELESS COMMUNICATION}

To further substantiate the improved spectral purity of the violet tunable SIL-ECDL system as a potential transmitter for future narrow-wavelength spaced OWC-WDM system, OOKNRZ transmission experiments are conducted on different tuned locked modes and compared with the FR case at different data rates, injection currents, and channel lengths. The parameters for the modulation are common for both the SIL and the FR cases, while the optical spectra with/without modulation are similar to the inset of Fig. 2(a). Fig. 5(a) shows the performance of two widely apart locked modes at 401.14 and $403.18 \mathrm{~nm}$ alongside the FR case for $0.4 \mathrm{~m} \mathrm{OWC}$ and at $35 \mathrm{~mA}(\sim 5.2 \mathrm{~V})$. At a lower data rate of $\leq 1.5 \mathrm{~Gb} / \mathrm{s}$, the performance of both the cases are similar to a measured BER of $\sim 3 \times 10^{-6}$. However, increasing the data rate to $\geq 1.75 \mathrm{~Gb} / \mathrm{s}$, a clear improvement in the BER of both SIL modes is noted compared to the FR counterpart. For instance, at $2.0 \mathrm{~Gb} / \mathrm{s}$, the shorter (longer) locked mode exhibited BER of $\sim 4 \times 10^{-4}$ $\left(\sim 8 \times 10^{-4}\right)$ with the wide-open eye, while the FR LD demonstrated $\sim 8 \times 10^{-3}$ that is above the forward error correction limit with an inferior open eye. This corresponds to $\sim 10$ times improvement in the performance of the system, which is ascribed to the reduced ASE noise and hence high SNR of the locked modes, exhibited by the SIL-ECDL system.

Moreover, similar performance improvement is also noted at a high injection current of $50 \mathrm{~mA}(\sim 5.6 \mathrm{~V})$ with an extended channel length of $0.8 \mathrm{~m}$, as depicted in Fig. 5(b). In this case, at $1.75(2.0) \mathrm{Gb} / \mathrm{s}$, the $401.141 \mathrm{~nm}$ SIL mode demonstrates a measured BER of $\sim 3 \times 10^{-3}\left(\sim 12.8 \times 10^{-3}\right)$ compared to the FR LD spectrum that exhibits $\sim 5 \times 10^{-3}\left(\sim 13 \times 10^{-3}\right)$. Nevertheless, with these demonstrations, the potential of tunable violet tunable SIL-ECDL system prospects are bright in the future for both, OWC as well as flexible wavelength light source in various cross-disciplinary field applications.

\section{CONCLUSION}

A prism-based SIL-ECDL system with a violet InGaN/GaN LD is demonstrated with a continuous wavelength tunability from 400.28 to $405.43 \mathrm{~nm}(5.15 \mathrm{~nm})$. A rigorous analysis of the system performance showed a mean SMSR and optical linewidth of $\sim 23 \mathrm{~dB}$ and $\sim 190 \mathrm{pm}$, respectively, while displaying high system stability. Furthermore, an error-free indoor free-space transmission based on OOK-NRZ modulation scheme on 0.4 and $0.8 \mathrm{~m}$ channel length, and under different injection currents, is achieved with a maximum
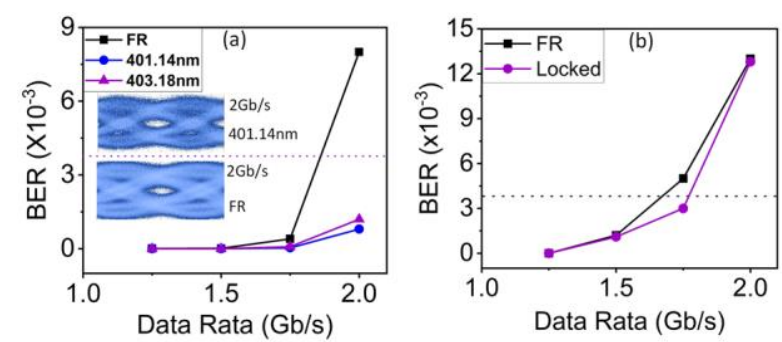

Fig. 5. BER versus the data rate of OOK-NRZ transmission experiments on (a) $0.4 \mathrm{~m}$ indoor free-space channel length at $35 \mathrm{~mA}$ and at two distinct tuned wavelengths of 401.14 and $403.18 \mathrm{~nm}$ (b) $0.8 \mathrm{~m}$ indoor free-space channel length at $50 \mathrm{~mA}$ and at a tuned wavelength of $401.14 \mathrm{~nm}$. The unlocked FR LD results are also shown. Insets of (a) are the eye diagrams.

data rate of $2.0 \mathrm{~Gb} / \mathrm{s}$ and $\sim 10$ times improved BER when compared to the FR LD case.

\section{REFERENCES}

[1] Y. C. Chi et al., "Violet Laser Diode Enables Lighting Communication," Sci. Rep., vol. 7, no. 1, pp. 1-11, 2017.

[2] C. Lee et al., "Gigabit-per-second white light-based visible light communication using near-ultraviolet laser diode and red-, green-, and blue-emitting phosphors," Opt. Express, vol. 25, no. 15, p. 17480, 2017.

[3] W. C. Wang, H. Y. Wang, and G. R. Lin, "Ultrahigh-speed violet laser diode based free-space optical communication beyond $25 \mathrm{Gbit} / \mathrm{s}$," Sci. Rep., vol. 8, no. 1, pp. 4-10, 2018.

[4] Y.-C. Chi et al., "450-nm GaN laser diode enables high-speed visible light communication with 9-Gbps QAM-OFDM," Opt. Express, vol. 23, no. 10 , p. $13051,2015$.

[5] T. C. Wu et al., "Tricolor R/G/B laser diode based eye-safe White lighting communication beyond 8 Gbit/s," Sci. Rep., vol. 7, no. 1, pp. 1$10,2017$.

[6] H. Park, D.-H. Kwon, and Y. Rhee, "High-resolution spectroscopy of Sm I performed with an extended-cavity violet diode laser," J. Opt. Soc. Am. B, vol. 21, no. 6, p. 1250, 2004.

[7] L. Mei, Z. Kong, and P. Guan, "Implementation of a violet Scheimpflug lidar system for atmospheric aerosol studies," Opt. Express, vol. 26, no. 6, p. A260, 2018.

[8] D. J. Lonsdale, A. P. Willis, and T. A. King, "Extended tuning and single-mode operation of anti-reflection-coated InGaN violet laser diode in a Littrow cavity," Meas. Sci. Technol., vol. 13, no. 4, pp. 488-493, 2002.

[9] T. Suski et al., "A pressure-tuned blue-violet InGaN/GaN laser diode grown on bulk GaN crystal," Appl. Phys. Lett., vol. 84, no. 8, pp. 1236$1238,2004$.

[10] M. H. M. Shamim et al., "Tunable self-injection locked green laser diode," Opt. Lett., vol. 43, no. 20, p. 4931, 2018.

[11] J. Ohtsubo, Semiconductor Lasers, Fourth. Springer International Publishing AG 2017, 2013.

[12] Y.-C. Chi et al., "Optical 16-QAM-52-OFDM transmission at 4 Gbit/s by directly modulating a coherently injection-locked colorless laser diode," Opt. Express, vol. 20, no. 18, p. 20071, 2012.

[13] M. H. M. Shamim et al., "Investigation of Self-Injection Locked Visible Laser Diodes for High Bit-Rate Visible Light Communication," IEEE Photonics J., vol. 10, no. 4, 2018.

[14] R. S. Conroy et al., "Characterization of an extended cavity violet diode laser," Opt. Commun., vol. 175, no. 1, pp. 185-188, 2000.

[15] K. Komorowska et al., "Tunable broad-area InGaN laser diodes in external cavity," Nov. In-pl. Semicond. Lasers VI, vol. 6485, p. 648502, 2007.

[16] C. Moser, L. Ho, and F. Havermeyer, "Self-aligned non-dispersive external cavity tunable laser," Opt. Express, vol. 16, no. 21, p. 16691, 2008.

[17] X. Q. Lv et al., "Tuning properties of external cavity violet semiconductor laser," Chinese Phys. Lett., vol. 30, no. 7, 2013.

[18] B. Li et al., "Studies on $405 \mathrm{~nm}$ blue-violet diode laser with external grating cavity," Nov. In-pl. Semicond. Lasers XV, vol. 9767, p. 97670E, 2016.

[19] K. Nakamura, I. Mizukoshi, and M. Hanawa, "Optical wireless transmission of $405 \mathrm{~nm}, 145 \mathrm{Gbit} / \mathrm{s}$ optical IM/DD-OFDM signals through a $48 \mathrm{~m}$ underwater channel," Opt. Express, vol. 23, no. 2, p. $1558,2015$. 